\title{
Selección de variedades de frijol común rojo (Phaseolus vulgaris L.), tolerantes a la sequía en diferentes condiciones de riego en campo Selection of varieties of common red bean (Phaseolus vulgaris L.), tolerant to drought in different irrigation conditions in the field
}

\section{Amalia Domínguez Suárez¹, Rodolfo Darias Rodríguez¹, Yordanys Martínez Dávalos¹, Maryla Sosa del Castillo¹, Daynet Sosa del Castillo²}

DOI. 10.21931/RB/2021.06.01.6

Resumen: El objetivo del presente trabajo fue seleccionar variedades de frijol rojo más tolerantes a la sequía, en condiciones experimentales de sequía, en Campo. Para ello se realizó un experimentos con cinco variedades de frijol común de color rojo (Buenaventura, CC 25-9 rojo, Velazco Largo, La Cuba 154 y Delicia 364), en diferentes condiciones de humedad del suelo. Se evaluaron indicadores fenológicos y de rendimiento, en condiciones diferentes de riego, cinco riegos (sequía) y 10 riegos (condiciones óptimas de humedad). Con los datos de rendimientos en las dos condiciones de humedad se calculó el porcentaje de pérdidas del rendimiento, los índices de intensidad de sequía (IIS) y susceptibilidad a la sequía (ISS). El análisis estadistico fue realizado utilizando el programa InfoStat versión 2011. Se llegó a la conclusión que las variedades Cuba Cueto 25-9 colorado, La Cuba 154 y Delicia 364 fueron las que tuvieron el comportamiento más tolerante en las condiciones de experimentación.

Palabras clave: frijol común, riego, tolerancia a sequía, rendimiento.

Abstract: The present work's objective was to select more tolerant varieties of red beans to the field's drought. It came right one experiment with five varieties of red-colored common beans (Buenaventura, CC 25-9 colorado, Velazco Largo, La Cuba 154, and Delicia 364) different conditions of soil water. Indicators of performance were evaluated in different irrigation conditions, 5 irrigations (drought), and 10 irrigations (optimal humidity conditions). With the yield data in the two conditions of humidity, the intensity of drought (IIS), drought resistance (ISS), and yield losses were calculated. The statistical analysis was accomplished using the program InfoStat version 2011. It took place to conclude that the studied varieties showed the most tolerant behavior in Cuba Cueto 25-9 Rojo, La Cuba 154 y Delicia 364.

Key words: Common bean, irrigation, tolerance to drought, yields.

\section{Introducción}

El frijol común (Phaseolus vulgaris L.) forma parte del grupo de las leguminosas comestibles, es importante no solo por sus propiedades nutricionales y culinarias, sino además por su presencia en los cinco continentes del mundo y su importancia para el desarrollo rural y social de muchas economías ${ }^{1}$. Este producto es un alimento tradicional en América Latina y el Caribe?

El cultivo del frijol (Phaseolus vulgaris L.) al igual que otros cultivos de importancia económica se afecta durante el crecimiento y desarrollo por factores bióticos y ambientales adversos (abiótico) como: precipitación, temperatura, humedad, luminosidad y mala distribución del área cultivada ${ }^{1}$. Así como factores edáficos que varían entre localidades como: topografía, profundidad del suelo y época de siembra que puede favorecer la aparición de plagas y enfermedades. Además influye la falta de cultivares adaptados al medio ambiente, incluso a los cambios climatológicos a nivel global, por lo que es importante contar indicadores morfológicos y agronómicos para caracterizar cultivares y líneas en diferentes épocas de cultivo ${ }^{3}$.

Se reporta en la literatura que el estrés por sequía, causado por la baja disponibilidad de agua en el suelo, modifica negativamente la productividad del frijol ${ }^{4,5}$. Esta puede ser más o menos afectada dependiendo de la intensidad y duración de la escasez de agua, de la rapidez con la cual se alcance dicha intensidad y además de la etapa fenológica en que el efecto ocurra, así como el pre acondicionamiento de la planta 6 .

Agunas de estas modificaciones se consideran respues- tas metabólicas que constituyen mecanismos de adaptación al déficit hídrico en las plantas ${ }^{7}$. Se destacan por su correlación positiva con la tolerancia a la sequía la longitud de la raíz y su relación con la producción de biomasa ${ }^{8}$, el índice de marchites y el contenido relativo de agua de las hojas ${ }^{9}$, tanto en la fase vegetativa como reproductiva.

En la fase reproductiva en frijol y otras leguminosas disminuye el rendimiento en mayor proporción, que cuando solo afecta la fase vegetativa ${ }^{10,11}$, lo que depende de la intensidad del estrés hídrico y la tolerancia del cultivar. por lo que varios autores han tenido en cuenta los indicadores fenológicos, la pérdida de rendimiento y el índice de susceptibilidad a la sequía, en estudios de evaluación y selección de variedades de frijol tolerantes a la sequía, ${ }^{6,12,13}$.

En nuestro país muchas de las áreas donde se produce fríjol, son cultivadas con un suministro de agua dependiente de la precipitacion. De ahí la importancia de seguir profundizando en el estudio de variedades comercializadas en Cuba. Este trabajo tuvo el objetivo de seleccionar cultivares tolerantes a la sequía, en condiciones de campo, con un rendimiento adecuado.

\section{Materiales y métodos}

El experimento se realizó en la finca "Sabanilla " de la CCS "victoria de Girón" que está enclavada en el municipio de

${ }^{1}$ Centro de Estudios Biotecnológicos, Facultad de Ciencias Agropecuarias, Universidad de Matanzas, Cuba.

${ }^{2}$ Escuela Superior Politécnica del Litoral (ESPOL), Guayaquil, Ecuador. 
Unión de Reyes, en el poblado Juan G. Gomez, en la provincia de Matanzas, Cuba, la cual cuenta con una extensión de 13,5 ha. El suelo es pardo Mullido Carbonatado ${ }^{14}$, con un contenido de materia orgánica entre medio y alto, el pH es neutro y el contenido de $\mathrm{P}_{2} \mathrm{O}_{5}$ fue de $1,45 \mathrm{mg} / 100 \mathrm{~g}$ y el de $\mathrm{K}_{2} \mathrm{O}$ de 24,75 $\mathrm{mg} / 100 \mathrm{~g}$, los cuales están dentro de los rangos permisibles para el cultivo del frijol, recomendando añadir materia orgánica y no fertilizante. Durante la duración del ensayo la temperatura media fue de $21,35 \stackrel{\circ}{C}$ y el promedio de precipitación de $8 \mathrm{~mm}$.

La investigación se realizó, en los meses de enero a marzo de 2018, las temperaturas (máxima, y mínimas) fueron variables, donde las temperaturas medias oscilaron entre 22 y 29 oC las temperaturas mínimas oscilaron entre 15 y $27 \stackrel{0}{ }$ C y las máximas entre 28 y $31 \stackrel{\circ}{\circ}$. Por otro lado, los valores de humedad relativa oscilaron entre 32 y $78 \%$. Ambos valores permisibles para el desarrollo del cultivo. El promedio de precipitaciones fluctúo entre 9-60 mm, según el centro Meteorológico Unión de Reyes.

Se utilizaron cinco variedades de frijol común de color rojo: Buenaventura, Velazco Largo, Cuba Cueto rojo (R), La Cuba154 y Delicia 364, todos suministrado por la empresa de semilla de Jovellanos, Matanzas.

El experimento se sembró el 20 diciembre del 2018. Se utilizó un diseño de bloque al azar, en surcos de $7 \mathrm{~m}$ de largo $X$ 0,60 m de ancho, en un área total de 0,033 ha, con tres repeticiones por variedad y tratamiento, con una densidad de 15-18 granos $\mathrm{m}^{-1}{ }^{15}$.

\section{Traramientos}

$\mathrm{T} 1$ : cultivares de frijol bajo riego (10 R)

T2: cultivares de frijol en condiciones de sequía (5R)

En el caso del ensayo con riego se aplicaron 10 riegos considerando los requerimientos hídrico según etapas de dedsarrollo del frijol, con una norma neta total promedio de $3500 \mathrm{~m}^{3}$ ha-1 $^{-15}$.

\section{Variables analizadas y metodología empleadas}

Se evaluaron indicadores fenológicos, de rendimiento y de productividad.

En la madurez fisiológica se cosecharon 10 plantas por tratamientos y repeticiones a las que determinaron el rendimiento en $t$ ha $^{-1}$. Además, registraron los datos fenológicos de días de inicio de floración (DIF), días de madurez fisiológica (DMF) e índice reproductivo (IR) según la ecuación ${ }^{12}$ :

IR $(\%)=1-(\mathrm{DPR} / \mathrm{DMF}){ }^{*} 100$

Donde:

$\mathrm{DPR}$ = días del período reproductivo (DMF-DIF)

Al alcanzar la madurez de la cosecha se recolectaron muestras de 2 metros por surcos de cada variedad y por condición de riego, lo que equivale a un área de $2,4 \mathrm{~m}^{2}$ para calcular los indicadores de productividad: número de vainas por plantas, número promedio de semillas por vaina, número de semillas por plantas y el peso de cien semillas (g). Se descar- taron los 0,5 m iniciales y finales para evitar el efecto borde y cabecera.

Para estimar la reducción del rendimiento por causa del estrés hídrico se aplicó la siguiente ecuación, Perdida de rendimiento $(P R)=1-(\operatorname{Re} / \operatorname{Rr}) \times 100$, donde:

$\mathrm{Re}=$ promedio general de rendimiento en sequía

$\mathrm{Rr}=$ promedio general de rendimiento en riego .

Para estimar la intensidad y el efecto de la sequía sobre el rendimiento, se determinó el Î́ndice de intensidad de sequía (IIS) mediante la siguiente ecuación ${ }^{12}$ :

IIS $=[1-(\mathrm{RS} / \mathrm{RRS})]$, donde:

$\mathrm{RS}=$ promedio general de rendimiento en sequía.

$\mathrm{RRS}=$ promedio general de rendimiento en riego.

El Índice de susceptibilidad a la sequía (ISS) para cada variedad fue determinado con la ecuación reportada por Chávez et al. $(2018)^{13}$

ISSi= [1-(RSi/RRSi)]/IIS, donde:

ISSi= Índice de susceptibilidad a sequía $(5 R)$ de la i-ésima variedad.

$\mathrm{RSi}=$ rendimiento promedio en sequía $(5 R)$ de la i-ésima variedad.

$\mathrm{RRSi}=$ rendimiento promedio en riego suplementario (10 $R)$ para la i-ésima variedad.

Para el cálculo de estos índices se utilizaron los valores de rendimientos obtenidos en cada repetición de sequía (5R) con su correspondiente repetición en riego (10 R).

La comparación de las medias para el análisis de los resultados se realizó a través de la prueba de Tukey, con una probabilidad de error de 5\%, mediante el programa InfoStat ${ }^{16}$.

\section{Resultados y discusión}

Al analizar el comportamiento de los indicadores fenológicos (la Tabla 1), se observan diferencias entre las variedades al comparar los resultados de las dos condiciones de riego, con un índice de intensidad de sequía de 0,24. En la condición de déficit hídrico $(5 R)$ todas las variedades adelantaron su floración, de tres a cuatro días, con respecto a su similar en condiciones óptimas de riego. La Cuba 154 y Buenaventura, fueron las que presentaron la mayor precocidad con respecto a la aparición de las flores (cuatro días antes), mientras que el resto de las variedades tuvieron sus primeras flores abiertas tres días antes.

En relación a los días a madurez fisiológica, para las plantas del tratamiento con riego oscilaron los valores entre $71 \mathrm{y}$ 67 días después de la siembra; mientras que en condiciones de sequía, fue entre 61 y 90 días, por lo que todas las plantas alcanzaron más temprano la madurez de cosecha en condiciones de sequía. La variedad Delicia fue la más precoz y mantuvo el índice reproductivo (IR) mayor (Tabla1).

El índice de intensidad de sequía (IIS), indica el grado de estrés al que fueron sometidos las variedades y la idoneidad de

\begin{tabular}{lllllll}
\hline & \multicolumn{2}{l}{ DF (días) } & \multicolumn{2}{l}{ DMF (dias) } & \multicolumn{2}{l}{ IR (\%) } \\
\hline Variedades & I0R & $5 \mathrm{R}$ & $10 \mathrm{R}$ & $5 \mathrm{R}$ & $10 \mathrm{R}$ & $5 \mathrm{R}$ \\
\hline Delicia 364 & 37 & 34 & 70 & 63 & 52 & 53 \\
Buenaventura & 33 & 29 & 69 & 64 & 47 & 46 \\
Velazco Largo & 31 & 28 & 67 & 61 & 46 & 45 \\
\hline La Cuba 156 & 40 & 36 & 69 & 67 & 42 & 49 \\
\hline Cuba Cueto R & 40 & 37 & 71 & 68 & 43 & 45 \\
\hline
\end{tabular}

Tabla 1. Variables fenológicas de las variedades bajo dos condiciones de riego en CCS "Victoria de Girón”, de enero a marzo 2019. 
las condiciones que se tuvieron en este estudio para la identificación de las variedades con resistencia a sequía. La variación presentada en el ciclo fenológico concuerda con lo reportados por otros autores con índice semejante de 0,2717. Así como otros que reportan IIS mayores de 0,5, caracterisíco de estado de sequía terminal), quienes consideran que la precocidad en la maduración es una característica muy valiosa en la adaptación del frijol común a sequía, ya que permite completar el llenado del grano con una mayor disponibilidad de agua con respecto a genotipos más tardíos ${ }^{13}$. El índice reproductivo de las variedades mostró respuestas diferentes entre ellas, se pueden considerar como las más adaptadas, a las condiciones experimentales, aquellas que combinan un índice reproductivo alto y un mayor período desde la floración hasta la madurez, como son La Cuba y Cuba Cueto R, lo que favorece un período amplio para la formación de órganos reproductivos, lo que favorece la formación de granos, por lo que los resultados obtenidos indican que las variedades de frijol estudiadas poseen entre otras características, plasticidad fenológica, ya que presentar una temprana floración y madurez lo que reduce el impacto negativo del estrés así que una de las formas de escape a la sequía se basa en un rápido desarrollo fenológico ${ }^{13,18}$.
Al analizar el comportamiento de los indicadores de rendimiento (peso de 100 semillas, número de vainas por plantas y número de semillas por vainas), en las dos condiciones de riego (Tabla 2), se pudo constatar que no semodificaron por igual todos los parametros estudiados, en las condiciones de sequía (5R), en las diferentes variedades. El peso de 100 semillas fue el indicador que presentó diferencia significativa, entre las medias de las dos condiciones de riego, en todas las variedades, lo que puede explicarse por el papel determinante del deficit hídrico en la producción de vainas y llenado de las mismas ${ }^{12}$. El número de semillas por vaina fue el que presentó menor afectación, seguido del número de vainas por plantas, que solo en la variedad Buenaventura hubo diferencia Aunque no hubo diferencia en el número de semillas por vainas, cuando se analiza la morfología de la semilla si se aprecia diferencia (Figura 1), lo que explica la diferencia en el peso de cien semillas. Esto concuerda con lo reportado en estudio realizado, con cuatro variedades de frijol negro, cultivada en condiciones de estrés, que también las vainas presentaron granos más pequños que las de su igual sin defícit hídrico y no hubo diferencia en el númerode semillas por vainas ${ }^{19}$.

\begin{tabular}{|c|c|c|c|c|c|c|}
\hline \multirow{2}{*}{$\underbrace{\text { Variedades }}_{\text {Riegos }}$} & \multicolumn{2}{|c|}{$\begin{array}{l}\text { Peso } 100 \text { Semillas } \\
\text { (g) }\end{array}$} & \multicolumn{2}{|c|}{$\begin{array}{l}\text { Número } \\
\text { Vainas/Plantas }\end{array}$} & \multicolumn{2}{|c|}{$\begin{array}{l}\text { Nủmero } \\
\text { Semillas/vainas }\end{array}$} \\
\hline & $10 \mathrm{R}$ & $5 \mathrm{R}$ & $10 \mathrm{R}$ & $5 R$ & $10 \mathrm{R}$ & $5 \mathrm{R}$ \\
\hline Delicia 364 & $30,62 \mathrm{a}$ & $26,88 \mathrm{~b}$ & $19,2 \mathrm{a}$ & $18,3 \mathrm{a}$ & $2,91 \mathrm{a}$ & $2,83 a$ \\
\hline Buenaventura & $32,66 \mathrm{a}$ & $28,42 \mathrm{~b}$ & $14 \mathrm{a}$ & 11,75 & $3,92 \mathrm{a}$ & $3,38 \mathrm{a}$ \\
\hline Velazco Largo & $42,96 \mathrm{a}$ & $39,41 \mathrm{~b}$ & $6,3 \mathrm{a}$ & 8,3 a & $3,57 \mathrm{a}$ & $3,49 \mathrm{a}$ \\
\hline Cuba Cueto rojo & 28,79 a & $25,33 \mathrm{~b}$ & 15,90 & 14,98 & $6,23 \mathrm{a}$ & $5.83 \mathrm{a}$ \\
\hline La Cuba 154 & $34,45 \mathrm{a}$ & $31,66 \mathrm{~b}$ & 15,23 & $14.51 \mathrm{a}$ & $5.5 \mathrm{a}$ & $4,9 \mathrm{a}$ \\
\hline
\end{tabular}

Tabla 2. Indicadores de rendimiento de las variedades estudiadas bajo diferentes condiciones de riego.
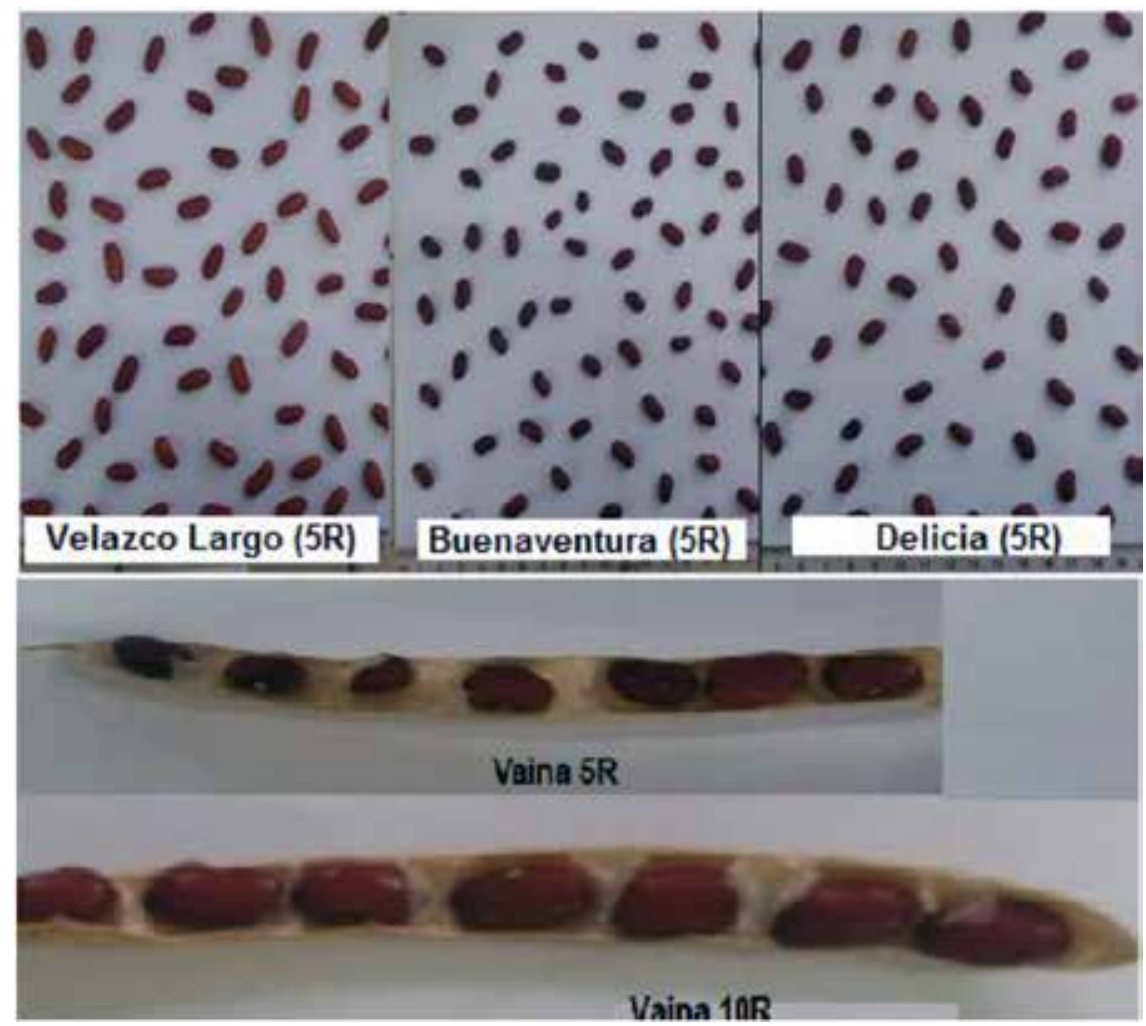

Figura 1. Ejemplo de la morfología de semillas cosechadas en condiciones de sequía (5R) y vainas en las dos condiciones de riego. 
Con los resultados obtenidos de los indicadores de rendimiento, en ambas condiciones de riego, se calculó la pérdida de rendimiento $(P R)$ de cada variedad (Figura 2). En genera, hubo una disminución en todos los indicadores estudiados: $15,3 \%$ vainas por plantas, $14,6 \%$ de semillas por vainas, $24,9 \%$ de semillas por plantas y $17,8 \%$ en el rendimiento total. Las variedades con los mayores porcentajes de pèrdida de rendimiento de número de vainas por plantas, número de semillas por vainas y número semillas por plantass, fueron Velazco Largo y Buenaventura. También la pérdida de rendimiento total, debido a la sequía, fue indudable en todas las variedades, pero fue más acentuada en las variedades Velazco Largo con 33,78 $\%$ y la de menor fue Delicia con solo un 6,5\%, seguido de Cuba mayor que uno, lo que evidencia menor tolerancia a la sequía. Por otra parte, Buenaventura presenta un ISS menor que uno pero su indice reproductivo (IR) no fue alto y tuvo un $20 \%$ de perdida del rendimiento.

Estos resultados corroboran el efecto negativo de la sequía, lo que ha sido reportado por diferentes autores. Los índices de selección utilizados permiten caracterizar el comportamiento de estos genotipos en diferentes condiciones de humedad en el suelo, aunque se sugiere la utilización combinada de por lo menos un índice de cada grupo con tal de combinar el alto potencial de rendimiento con la tolerancia a la sequía. ya que las variedades que crecieron con menos disponibilidad de agua, tuvieron menos rendimiento. No todas las variedades respondieron de igual forma, presentaron un índice

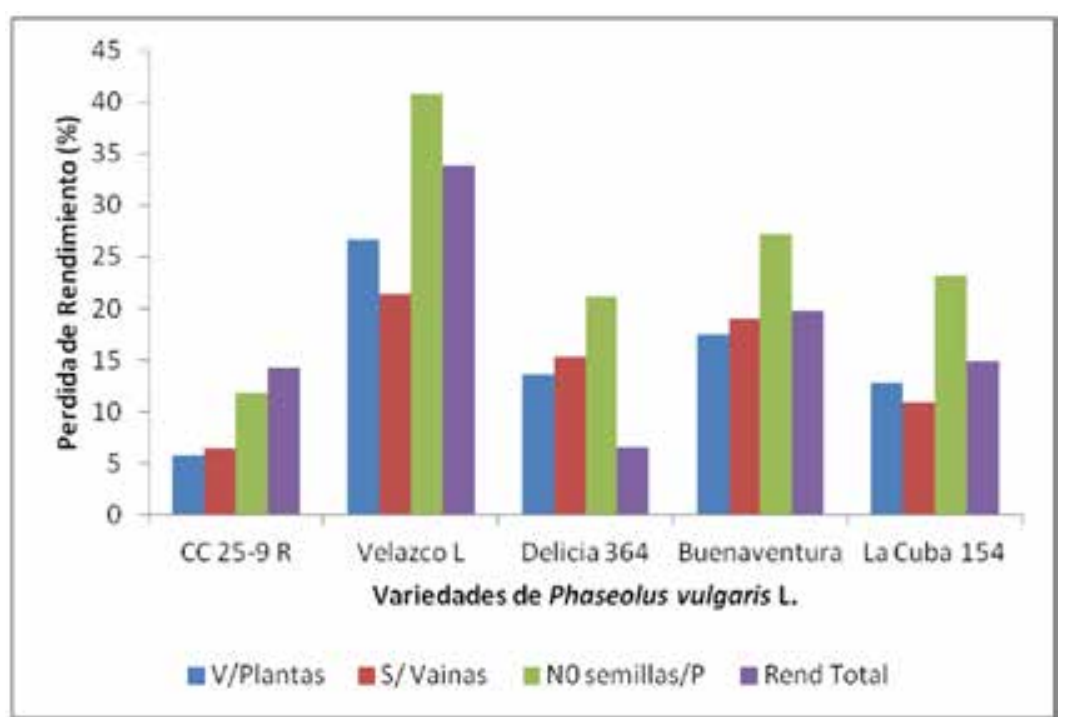

Figura 2. Porcentaje de reducción de los indicadores de rendimiento evaluados, bajo condiciones de sequía, enero-marzo 2019. Leyenda: NV/plantas: número de vainas por plantas; NS/vainas: número de semillas por vainas; NS/plantas. Número semillas por plantas y Rend Total: rendimiento total.

En trabajos realizados en campo, en condiciones de sequía experimental (cuatro riego) se reporta una disminución del rendimiento total por hectárea hasta un $48,59 \%$ en este tipo de leguminosa ${ }^{6}$.

También en experimento realizado con frijol Caupí se reporta disminución de $57,72 \%$, del rendimiento de grano por planta, 49,40\%, del número de vainas por planta, 32,07\% del número de semillas por vaina ${ }^{7}$.

Los resultados de índices de susceptibilidad a la sequía (ISS) para los genotipos estudiados estuvieron entre 0,27 y 1,77 (Tabla 3).

Las variedades Delicia, Cuba Cueto R y La Cuba 154 presentaron una menor reducción del rendimiento, simultáneamente a un menor índice de susceptibilidad a la sequía (ISS), lo que expresa una mayor tolerancia al déficit hídrico. Sin embargo, Velazco Largo disminuyó su rendimiento y presento un ISS

de estrés que varía en correspondencia a la tolerancia a la sequía que caracteriza a la variedad $d^{5,11,13}$.

La respuesta de las plantas a este estrés ambiental depende de varios factores incluyendo el genotipo, la etapa del desarrollo de la planta, la longitud y la severidad del estrés aplicado ${ }^{11,12}$. Por otra parte, las características del suelo pueden influir en dichos resultados, de ahí la importancia de evaluar el comportamiento productivo de las variedades en cada localidad $^{14}$

Como consecuencia de los cambios climáticos los períodos de sequía dentro del ciclo de cultivo del frijol cada vez es más son más intensos. Debido a esta razón, es importante enfocar el mejoramiento del cultivo por resistencia a sequía hacia genotipos que tengan rendimientos altos, tanto en una condición de estrés como cuando no la haya. Bajo este precepto las variedades La Cuba y Cuba Cueto R fueron las mejores, con

\begin{tabular}{|c|c|c|c|c|}
\hline \multirow[t]{2}{*}{ Variedades } & Rendimiento & $\left(t h^{-1}\right)$ & \multirow[t]{2}{*}{ ISS } & \multirow[t]{2}{*}{ Respuesta a la sequia } \\
\hline & I0R & $5 R$ & & \\
\hline Delicia & $0.86 \mathrm{a}$ & $0,81 \mathrm{a}$ & 0.66 & Tolerante \\
\hline Buenaventura & $0,99 \mathrm{a}$ & $0.79 \mathrm{~b}$ & 0,97 & Medianamente Tolerante \\
\hline La Cuba 154 & $1,9 \mathrm{a}$ & $1,61 a b$ & 0,27 & Tolerante \\
\hline Velazco L. & $1,16 \mathrm{a}$ & $0,77 b$ & 1,77 & Menos Tolerante \\
\hline Cuba Cueto $\mathbf{R}$ & $1,9 a$ & $1,63 \mathrm{ab}$ & 0.83 & Tolerante \\
\hline
\end{tabular}

Tabla 3. Respuesta de las variedades a la sequía según su rendimiento e ISS. 
excelentes rendimientos en ambos ambientes y que podrían representar una solución para las zonas propensas a sufrir sequía o para tener en cuenta a la hora de sembrar en el periodo poco lluvioso (enero-marzo). También la variedad Delicia, aunque no tuvo alto rendimiento en ninguna de las dos condiciones pero entre ellas no hubo diferencia.

El índice de susceptibilidad a la sequía (ISS) puede ser considerado como un criterio aceptable para discriminar variedades, bajo condiciones de estrés hídrico. No obstante, se deben tener en cuenta otras características; ya que puede darse el caso que las variedades con mayor tolerancia a la sequía (menor ISS), no necesariamente sean las más productoras en esas condiciones, pero si los que menos reducen su rendimiento $0^{5,17}$.

\section{Conclusiones}

Las variedades Cuba Cueto 25-9 colorado, La Cuba 154 y Delicia 364 presentaron los indices de intensidad de sequía (ISS) menores a 1, por lo que se consideran como tolerantes a la sequìa, recomendàndose su empleo en la localidad analizada y en la epoca de siembra que se corresponde con los periodos poco lluviosos en Cuba.

La variedad Velazco Largo es la màs afectada por la sequia lo que se corresponde con su ISS y su rendimiento, por lo tanto, para la localidades en condiciones de secano no se recomienda su cultivo en epoca de siembra de pocas lluvias.

El déficit hídrico a pesar de que no afectó el nùmero de vainas por planta y de semillas por vaina, sì afectó la morfología y peso de las semillas.

\section{Referencias bibliográficas}

1. Hernández, J.C.; Chaves, N.F.; Arayar, Y.; Beebe, S. Diquís", variedad de frijol común rojo brillante. Agronomía Costarricense. 2018; 42(1): 127-136. ISSN 0377-9424.

2. FAO (Organización Mundial de la Alimentación). El futuro de la alimentación y la agricultura. Tendencias y Desafíos (2017).Disponible:<www.fao.org/3/a-i6583e> [Consultado: 16 de marzo 2020].

3. Martirena-Ramírez, A.; Veitía, N.; García, LR.; Collado, R.; Torres, D.; Rivero Quintana, L.; Ramírez- López, M. Caracterización morfológica de líneas de Phaseolus vulgaris L. en casa de cultivo. Biotecnología Vegetal. 2017;17(3):191-20

4. Polania, J.A.; Rao, I.M.; Mejía, S.; Beebe, S.E.; Cajiao, C. Características morfofisiológicas del frijol común (Phaseolus vulgaris L.) relacionadas con la adaptación a sequía. Acta Agron. 2012; 61(3): 197-206.

5. Estrada, W.; Chávez, L.; Jerez, E.; Nápoles, M.C.; Sosa, A.; Maceo Y.C.; Cordoví, C. Respuesta de cultivares de frijol (Phaseolus vulgaris L.) a la sequía utilizando diferentes índices de selección. Cultivos tropicales. 2016; 37(3):79-84. DOl: 10.13140/ RG.2.1.5181.2082

6. Domínguez, A.; Darias, R.; Martínez, Y.; Alfonso, E. Tolerancia al déficit hídrico de variedades de frijol común en condiciones de sequía experimental, en campo. Revista Centro Agrícola. 2019a; 46 (3): 22-29. ISSN: 0253-5785 ISSN on line: 2072-2001.
7. Cardona, C., Jarma A.J.; Araméndiz, H.; Peña, M.; Vergara, C. Respuesta fisiológicas y bioquímicas del frijol caupi (Vigna unguilata L. Walp) bajo déficit hídrico". Rev. Colomb. Cienc. Hortic. 2014; 8(2):250-261.

8. Martirena-Ramírez, A.; Veitía, N.; García, L.R.; Collado, R.; Torres, D.; Rivero- Quintana, L.; Ramírez- López, M. Respuesta de líneas de Phaseolus vulgaris L. en época de siembra tardía. Biotecnología Vegetal. 2018; 18(2): 117-123.

9. Domínguez, A.; Pérez, Y.; Alemán, S.; Sosa, M.; Fuentes, L.; Darias, R. (et al). Respuesta de cultivares de Phaseolus vulgaris L. al estrés por sequía. Biotecnología Vegetal. 2014; 14 (1): 29 - 36. ISSN 2074-864.

10.Polón-Pérez, R.; Ruiz-Sánchez, M.; Miranda-Caballero, A.; Ramírez-Arrebato, M.A. Efectos del estrés hídrico sobre el rendimiento de los granos del frijol (Phaseolus vulgaris L.). Revista Ciencias Técnicas Agropecuarias. 2017; 26(1): 66-70). ISSN -1010-2760, E-ISSN: 2071-0054.

11. Polón, R.; Miranda, A.; Ramírez, M.A.; López, L.A. Efectos del estrés de agua sobre el rendimiento de granos en la fase vegetativa en el cultivo del frijol (Phaseolus vulgaris L). Revista Ciencias Técnicas Agropecuarias. 2014; 23: 4. ISSN -1010-2760, RNPS0111.

12. Boicet, T.; Secada, Y.; Chaveco, O.; Bouder, A.; Gómez, Y.; Meriño, Y. (et al). Respuesta a la sequía de genotipos de frijol común utilizando diferentes índices de selección. Centro Agrícola. 2011; 38 (4): 69-73.

13. Chaves-Barrantes N.F.; Polanía J, A.; Muñoz-Perea C.G.; Rao I.M.; Beebe, S.E. Caracterización fenotípica por resistencia a sequía terminal de germoplasma de frijol común. Agron. Mesoam. 2018; 29(1):1-17. ISSN 2215-3608. doi:10.15517/ma.v29i1. 27618.

14. Hernández, A.; Pérez, J.M.; Bosch, D.; Castro, N. Clasificación de los suelos de Cuba. Publicado en formato digital. Ediciones INCA. La Habana, Cuba. 2015; 53-57. ISBN: 978-959-7023-77-7

15. Faure, B.; Benitez, R.J.; León, N.; Chaveco, O.; Rodríguez, O. Guía técnica para el cultivo del frijol común (Phaseolus Vulgaris L). Instituto de Inestigaciones de Grano. Editora Agroecológica. La Habana, 2012.

16. Di Rienzo, J.A.; Balzarini, M.; Casanoves, F.; González, L.; Tablada, M.; Robledo, C.W. InfoStat/ profesional versión 1.1.; 2011. Facultad de Ciencias Agropecuarias de la Universidad Nacional de Córdoba. Córdoba.

17. Meriño, H.Y.; Boudet, A.A.; Boicet, F.T.; Amado, B.E.; Palacio, A.J.; Castillo, R.O. "Rendimiento y tolerancia a la sequía de seis variedades de frijol común (Phaseolus vulgaris L.) en condiciones de campo". Centro Agrícola. 2015; 42(1): 69-74, ISSN: 2072-2001.

18. Pedroza-Sandoval, A.; Trejo-Calzada, R.; Sánchez-Cohen, I.; Samaniego-Gaxiola, JA., Yánez-Chávez, LG. Evaluación de tres variedades de frijol pinto bajo riego y sequía en Durango, México. Agronomía Mesoamericana. 2016; 27(1):167-76. doi:10.15517/ am. v27i1.21896.

19. Domínguez, A.; Darias, R.; Martínez, Y.; Bello, A.. Comportamiento productivo de variedades de frijol (negro) en condiciones de sequía en CCS Sabino Pupo. Revista Avanzada Científica. 2019b; 22(2):18-28. ISSN 1029-3450.

Received: 10 Octubre 2020 Accepted: 7 enero 2021 